Bull. Korean Math. Soc. 52 (2015), No. 6, pp. 1805-1818

http://dx.doi.org/10.4134/BKMS.2015.52.6.1805

\title{
ON CERTAIN GENERALIZED $q$-INTEGRAL OPERATORS OF ANALYTIC FUNCTIONS
}

\author{
Sunil Dutt Purohit and Kuppathai Appasamy Selvakumaran
}

\begin{abstract}
In this article, we first consider a linear multiplier fractional $q$-differintegral operator and then use it to define new subclasses of $p$ valent analytic functions in the open unit disk $\mathcal{U}$. An attempt has also been made to obtain two new $q$-integral operators and study their sufficient conditions on some classes of analytic functions. We also point out that the operators and classes presented here, being of general character, are easily reducible to yield many diverse new and known operators and function classes.
\end{abstract}

\section{Introduction and preliminaries}

Recently, S. D. Purohit and R. K. Raina [18], [19], [20] and [21] have used the fractional $q$-calculus operators in investigating certain subclasses of functions which are analytic in the open unit disk $\mathcal{U}$. S. D. Purohit [17] also studied similar work and considered new classes of multivalently analytic functions in the open unit disk. The aim of this paper is to consider a linear multiplier fractional $q$-differintegral operator and to define certain new subclasses of functions which are $p$-valent and analytic in the open unit disk. Among the results derived include, two new $q$-integral operators and their sufficient condition inequalities for the subclasses defined and introduced below. Special cases of the main results are also mentioned.

Let $\mathcal{A}_{p}$ denote the class of functions $f(z)$ of the form

$$
f(z)=z^{p}+\sum_{n=p+1}^{\infty} a_{n} z^{n}, \quad(p \in \mathbb{N}=\{1,2,3, \ldots\}),
$$

which are analytic and $p$-valent in the open unit disk $\mathcal{U}=\{z \in \mathbb{C}:|z|<1\}$. A function $f \in \mathcal{A}_{p}$ is said to be $p$-valently starlike of order $\alpha(0 \leq \alpha<p)$ if and only if

$$
\Re\left\{\frac{z f^{\prime}(z)}{f(z)}\right\}>\alpha, \quad(z \in \mathcal{U}),
$$

Received February 17, 2014; Revised September 9, 2015

2010 Mathematics Subject Classification. 30C45, 26 A33.

Key words and phrases. analytic functions, $q$-integral operators, fractional $q$-calculus operators, $p$-valently starlike and $p$-valently convex functions of complex order. 
where the symbol $\Re\{f(z)\}$ denotes the real part of the function $f(z)$. We denote by $\mathcal{S}_{p}^{*}(\alpha)$ the class of all such functions. On the other hand, a function $f \in \mathcal{A}_{p}$ is said to be in the class $\mathcal{C}_{p}(\alpha)$ of $p$-valently convex of order $\alpha(0 \leq \alpha<$ $p$ ) if and only if

$$
\Re\left\{1+\frac{z f^{\prime \prime}(z)}{f^{\prime}(z)}\right\}>\alpha, \quad(z \in \mathcal{U}) .
$$

Note that $\mathcal{S}_{p}^{*}(0)=\mathcal{S}_{p}^{*}$ and $\mathcal{C}_{p}(0)=C_{p}$ are, respectively, the classes of $p$ valently starlike, and $p$-valently convex functions in $\mathcal{U}$. Also, we note that $\mathcal{S}_{1}^{*}(0)=\mathcal{S}^{*}$ and $\mathcal{C}_{1}(0)=\mathcal{C}$ are, respectively, the usual classes of starlike and convex functions in $\mathcal{U}$. A function $f \in \mathcal{A}_{p}$ is said to be in the class $\mathcal{S}_{p}(b, \alpha)$ of $p$-valently starlike of complex order $b(b \in \mathbb{C}-\{0\})$ and type $\alpha(0 \leq \alpha<p)$ if it satisfies

$$
\Re\left\{p+\frac{1}{b}\left(\frac{z f^{\prime}(z)}{f(z)}-p\right)\right\}>\alpha, \quad(z \in \mathcal{U}) .
$$

Furthermore, a function $f \in \mathcal{A}_{p}$ is said to be in the class $\mathcal{C}_{p}(b, \alpha)$ of $p$-valently convex of complex order $b(b \in \mathbb{C}-\{0\})$ and type $\alpha(0 \leq \alpha<p)$ if it satisfies

$$
\Re\left\{p+\frac{1}{b} \frac{z f^{\prime \prime}(z)}{f^{\prime}(z)}\right\}>\alpha, \quad(z \in \mathcal{U}) .
$$

Note that $\mathcal{S}_{p}(b, 0)=\mathcal{S}_{p}^{*}(b)$, the class of starlike functions of complex order $b(b \in \mathbb{C}-\{0\})$ and $\mathcal{C}_{p}(b, 0)=\mathcal{C}_{p}(b)$, the class of convex functions of complex order $b(b \in \mathbb{C}-\{0\})$. The class $\mathcal{S}_{p}^{*}(b)$ was studied by M. A. Nasr and M. K. Aouf [14], while the class $\mathcal{C}_{p}(b)$ was considered earlier by P. Wiatrowski [24].

For the convenience of the reader, we now give some basic definitions and related details of $q$-calculus which are used in the sequel:

For any complex number $\alpha$ the $q$-shifted factorials are defined as

$$
(\alpha ; q)_{0}=1, \quad(\alpha ; q)_{n}=\prod_{k=0}^{n-1}\left(1-\alpha q^{k}\right), \quad n \in \mathbb{N},
$$

and in terms of the basic analogue of the gamma function

$$
\left(q^{\alpha} ; q\right)_{n}=\frac{\Gamma_{q}(\alpha+n)(1-q)^{n}}{\Gamma_{q}(\alpha)}, \quad(n>0),
$$

where the $q$-gamma function is defined by

$$
\Gamma_{q}(x)=\frac{(q, q)_{\infty}(1-q)^{1-x}}{\left(q^{x} ; q\right)_{\infty}}, \quad(0<q<1) .
$$

If $|q|<1$, the definition (1.2) remains meaningful for $n=\infty$ as a convergent infinite product

$$
(\alpha ; q)_{\infty}=\prod_{j=0}^{\infty}\left(1-\alpha q^{j}\right)
$$

In view of the relation

$$
\lim _{q \rightarrow 1^{-}} \frac{\left(q^{\alpha} ; q\right)_{n}}{(1-q)^{n}}=(\alpha)_{n}
$$


we observe that the $q$-shifted factorial (1.2) reduces to the familiar Pochhammer symbol $(\alpha)_{n}$, where $(\alpha)_{n}=\alpha(\alpha+1) \cdots(\alpha+n-1)$.

It may be noted that the $q$-Gauss hypergeometric function ${ }_{2} \Phi_{1}[-]$ (see Gasper and Rahman [12, p. 3, eqn.(1.2.14)]) is defined by

$$
{ }_{2} \Phi_{1}[\alpha, \beta ; \gamma ; q, z]=\sum_{n=0}^{\infty} \frac{(\alpha ; q)_{n}(\beta ; q)_{n}}{(\gamma ; q)_{n}(q ; q)_{n}} z^{n}(|q|<1,|z|<1),
$$

and as a special case of the above series for $\gamma=\beta$, we have

$$
{ }_{1} \Phi_{0}[\alpha ;-; q, z]=\sum_{n=0}^{\infty} \frac{(\alpha ; q)_{n}}{(q ; q)_{n}} z^{n}(|q|<1,|z|<1) .
$$

Also, the $q$-derivative and $q$-integral of a function on a subset of $\mathbb{C}$ are, respectively, given by (see [12] pp. 19-22)

and

$$
D_{q} f(z)=\frac{f(z)-f(z q)}{(1-q) z}, \quad(z \neq 0, q \neq 0)
$$

$$
\int_{0}^{z} f(t) d_{q} t=z(1-q) \sum_{k=0}^{\infty} q^{k} f\left(z q^{k}\right)
$$

Therefore, the $q$-derivative of $f(z)=z^{n}$, where $n$ is a positive integer is given by

where

$$
D_{q} z^{n}=\frac{z^{n}-(z q)^{n}}{(1-q) z}=[n]_{q} z^{n-1}
$$

$$
[n]_{q}=\frac{1-q^{n}}{1-q}=q^{n-1}+\cdots+1
$$

and is called the $q$-analogue of $n$. As $q \rightarrow 1$, we have $[n]_{q}=q^{n-1}+\cdots+1 \rightarrow$ $1+\cdots+1=n$.

In the following, we define the fractional $q$-calculus operators of a complexvalued function $f(z)$, which were recently studied by S. D. Purohit and R. K. Raina [18].

Definition 1.1 (Fractional $q$-integral operator). The fractional $q$-integral operator $I_{q, z}^{\delta}$ of a function $f(z)$ of order $\delta$ is defined by

$$
I_{q, z}^{\delta} f(z) \equiv D_{q, z}^{-\delta} f(z)=\frac{1}{\Gamma_{q}(\delta)} \int_{0}^{z}(z-t q)_{\delta-1} f(t) d_{q} t, \quad(\delta>0),
$$

where $f(z)$ is analytic in a simply connected region of the z-plane containing the origin and the $q$-binomial function $(z-t q)_{\delta-1}$ is given by

$$
(z-t q)_{\delta-1}=z^{\delta-1} \Phi_{0}\left[q^{-\delta+1} ;-; q, t q^{\delta} / z\right]
$$

The series ${ }_{1} \Phi_{0}[\delta ;-; q, z]$ is single valued when $|\arg (z)|<\pi$ and $|z|<1$ (see for details [12], pp. 104-106), therefore, the function $(z-t q)_{\delta-1}$ in (1.3) is single valued when $\left|\arg \left(-t q^{\delta} / z\right)\right|<\pi,\left|t q^{\delta} / z\right|<1$ and $|\arg (z)|<\pi$. 
Definition 1.2 (Fractional $q$-derivative operator). The fractional $q$-derivative operator $D_{q, z}^{\delta}$ of a function $f(z)$ of order $\delta$ is defined by

$D_{q, z}^{\delta} f(z) \equiv D_{q, z} I_{q, z}^{1-\delta} f(z)=\frac{1}{\Gamma_{q}(1-\delta)} D_{q, z} \int_{0}^{z}(z-t q)_{-\delta} f(t) d_{q} t, \quad(0 \leq \delta<1)$, where $f(z)$ is suitably constrained and the multiplicity of $(z-t q)_{-}$is removed as in Definition 1.1.

Definition 1.3 (Extended fractional $q$-derivative operator). Under the hypotheses of Definition 1.2, the fractional $q$-derivative for a function $f(z)$ of order $\delta$ is defined by

$$
D_{q, z}^{\delta} f(z)=D_{q, z}^{m} I_{q, z}^{m-\delta} f(z),
$$

where $m-1 \leq \delta<1, m \in \mathbb{N}_{0}=\mathbb{N} \cup\{0\}$, and $\mathbb{N}$ denotes the set of natural numbers.

Remark 1.1. It follows from Definition 1.2 that

$$
D_{q, z}^{\delta} z^{n}=\frac{\Gamma_{q}(n+1)}{\Gamma_{q}(n+1-\delta)} z^{n-\delta} \quad(\delta \geq 0, \text { and } n>-1) .
$$

Using $D_{q, z}^{\delta}$, we define a $q$-differintegral operator $\Omega_{q, p}^{\delta}: \mathcal{A}_{p} \longrightarrow \mathcal{A}_{p}$, as follows:

$$
\begin{aligned}
\Omega_{q, p}^{\delta} f(z) & =\frac{\Gamma_{q}(p+1-\delta)}{\Gamma_{q}(p+1)} z^{\delta} D_{q, z}^{\delta} f(z) \\
& =z^{p}+\sum_{n=p+1}^{\infty} \frac{\Gamma_{q}(p+1-\delta) \Gamma_{q}(n+1)}{\Gamma_{q}(p+1) \Gamma_{q}(n+1-\delta)} a_{n} z^{n}, \\
& (\delta<p+1 ; n \in \mathbb{N} ; 0<q<1 ; z \in \mathcal{U}),
\end{aligned}
$$

where $D_{q, z}^{\delta} f(z)$ in (1.4) represents, respectively, a fractional $q$-integral of $f(z)$ of order $\delta$ when $-\infty<\delta<0$ and a fractional $q$-derivative of $f(z)$ of order $\delta$ when $0 \leq \delta<p+1$. Here we note that $\Omega_{q, p}^{0} f(z)=f(z)$.

Recently, K. A. Selvakumaran et al. [23] defined the linear multiplier fractional $q$-differintegral operator $\mathscr{D}_{q, p, \lambda}^{\delta, m}$ as follows:

$$
\begin{aligned}
\mathscr{D}_{q, p, \lambda}^{\delta, 0} f(z) & =f(z), \\
\mathscr{D}_{q, p, \lambda}^{\delta, 1} f(z) & =(1-\lambda) \Omega_{q, p}^{\delta} f(z)+\frac{\lambda z}{[p]_{q}} D_{q}\left(\Omega_{q, p}^{\delta} f(z)\right), \quad(\lambda \geq 0), \\
\mathscr{D}_{q, p, \lambda}^{\delta, 2} f(z) & =\mathscr{D}_{q, p, \lambda}^{\delta, 1}\left(\mathscr{D}_{q, p, \lambda}^{\delta, 1} f(z)\right), \\
& \vdots \\
\mathscr{D}_{q, p, \lambda}^{\delta, m} f(z) & =\mathscr{D}_{q, p, \lambda}^{\delta, 1}\left(\mathscr{D}_{q, p, \lambda}^{\delta, m-1} f(z)\right), \quad m \in \mathbb{N} .
\end{aligned}
$$

If $f(z) \in \mathcal{A}_{p}$ is given by (1.1), then by (1.5), we have

$$
\mathscr{D}_{q, p, \lambda}^{\delta, m} f(z)=z^{p}+\sum_{n=p+1}^{\infty}\left(\frac{\Gamma_{q}(p+1-\delta) \Gamma_{q}(n+1)}{\Gamma_{q}(p+1) \Gamma_{q}(n+1-\delta)}\left[1-\lambda+\frac{[n]_{q}}{[p]_{q}} \lambda\right]\right)^{m} a_{n} z^{n} .
$$


It can be seen that, by specializing the parameters the operator $\mathscr{D}_{q, p, \lambda}^{\delta, m}$ reduces to many known and new integral and differential operators. In particular, when $\delta=0, p=1$ and $q \rightarrow 1$ the operator $\mathscr{D}_{q, p, \lambda}^{\delta, m}$ reduces to the operator introduced by F. AL-Oboudi [2] and if $\delta=0, p=1, \lambda=1, q \rightarrow 1$ it reduces to the operator introduced by G. S. Sălăgean [22].

The $q$-analogues to the function classes $\mathcal{S}_{p}(b, \alpha)$ and $\mathcal{C}_{p}(b, \alpha)$ are given as follows:

A function $f \in \mathcal{A}_{p}$ is said to be in the class $\mathcal{S}_{q, p}(b, \alpha)$ of $p$-valently starlike of complex order $b(b \in \mathbb{C}-\{0\})$ and type $\alpha(0 \leq \alpha<p)$ with respect to $q$-differentiation if it satisfies

$$
\Re\left\{[p]_{q}+\frac{1}{b}\left(\frac{z D_{q}(f(z))}{f(z)}-[p]_{q}\right)\right\}>\alpha, \quad(z \in \mathcal{U}) .
$$

In addition, a function $f \in \mathcal{A}_{p}$ is said to be in the class $\mathcal{C}_{q, p}(b, \alpha)$ of $p$-valently convex of complex order $b(b \in \mathbb{C}-\{0\})$ and type $\alpha(0 \leq \alpha<p)$ with respect to $q$-differentiation if it satisfies

$$
\Re\left\{[p]_{q}+\frac{1}{b} \frac{z D_{q}^{2}(f(z))}{D_{q}(f(z))}\right\}>\alpha, \quad(z \in \mathcal{U}) .
$$

By using the operator $\mathscr{D}_{q, p, \lambda}^{\delta, m} f(z)$ defined by (1.5) and $q$-differentiation, we introduce two new subclasses of analytic functions $\mathcal{S}_{q, p, \lambda}^{\delta, m}(b, \alpha)$ and $\mathcal{C}_{q, p, \lambda}^{\delta, m}(b, \alpha)$ as follows:

A function $f(z) \in \mathcal{A}_{p}$ is said to be in the class $\mathcal{S}_{q, p, \lambda}^{\delta, m}(b, \alpha)$ if and only if

$\Re\left\{[p]_{q}+\frac{1}{b}\left(\frac{z D_{q}\left(\mathscr{D}_{q, p, \lambda}^{\delta, m} f(z)\right)}{\mathscr{D}_{q, p, \lambda}^{\delta, m} f(z)}-[p]_{q}\right)\right\}>\alpha, \quad(\alpha \in[0, p), b \in \mathbb{C}-\{0\}, z \in \mathcal{U})$.

Furthermore, a function $f(z) \in \mathcal{A}_{p}$ is said to be in the class $\mathcal{C}_{q, p, \lambda}^{\delta, m}(b, \alpha)$ if and only if

$$
\Re\left\{[p]_{q}+\frac{1}{b}\left(\frac{z D_{q}^{2}\left(\mathscr{D}_{q, p, \lambda}^{\delta, m} f(z)\right)}{D_{q}\left(\mathscr{D}_{q, p, \lambda}^{\delta, m} f(z)\right)}\right)\right\}>\alpha, \quad(\alpha \in[0, p), b \in \mathbb{C}-\{0\}, z \in \mathcal{U}) .
$$

It is interesting to note that, the classes $\mathcal{S}_{q, p, \lambda}^{\delta, m}(b, \alpha)$ and $\mathcal{C}_{q, p, \lambda}^{\delta, m}(b, \alpha)$ generalize several well known subclasses of analytic functions. For instance, when $p=$ $1, m=0$ and $b=1$ the class $\mathcal{S}_{q, p, \lambda}^{\delta, m}(b, \alpha)$ reduces to $\mathcal{S}_{q}^{*}(\alpha)$ the class of starlike functions with respect to $q$ differentiation of order $\alpha(0 \leq \alpha<1)$ and the class $\mathcal{C}_{q, p, \lambda}^{\delta, m}(b, \alpha)$ reduces to $\mathcal{C}_{q}(\alpha)$ the class of convex functions with respect to $q$ differentiation of order $\alpha(0 \leq \alpha<1)$, for details we refer to [23]. Also, the following cases are worthy of note here. If $q \rightarrow 1$, then

1. $\mathcal{S}_{1,1, \lambda}^{\delta, 0}(b, \alpha)=\mathcal{S}_{\alpha}^{*}(b)$ and $\mathcal{S}_{1,1,1}^{0,1}(b, \alpha)=\mathcal{C}_{1,1, \lambda}^{\delta, 0}(b, \alpha)=\mathcal{C}_{\alpha}(b)$ (see $\left.[10]\right)$, 
2. $\mathcal{S}_{1,1, \lambda}^{\delta, 0}(b, 0)=\mathcal{S}^{*}(b)\left(\right.$ see [14]) and $\mathcal{S}_{1,1,1}^{0,1}(b, 0)=\mathcal{C}_{1,1, \lambda}^{\delta, 0}(b, 0)=\mathcal{C}(b)$ (see $[24])$,

3. $\mathcal{S}_{1,1, \lambda}^{\delta, 0}\left(\cos \beta e^{-i \beta}, \alpha\right)=\mathcal{S}_{\alpha}^{\beta},(|\beta|<\pi / 2,0 \leq \alpha<1)$, the class of $\beta$-spirallike functions of order $\alpha$ (see [13]),

4. $\mathcal{C}_{1,1, \lambda}^{\delta, 0}\left(\cos \beta e^{-i \beta}, \alpha\right)=\mathcal{C}_{\alpha}^{\beta},(|\beta|<\pi / 2,0 \leq \alpha<1)$, the class of $\beta$ Robertson functions of order $\alpha$ (see [9]).

Using the operator $\mathscr{D}_{q, p, \lambda}^{\delta, m}, \mathrm{~K}$. A. Selvakumaran et al. in [23] introduced the following $p$-valent $q$-integral operators.

Definition 1.4. Let $m=\left(m_{1}, m_{2}, \ldots, m_{n}\right) \in \mathbb{N}_{0}^{n}, \gamma=\left(\gamma_{1}, \gamma_{2}, \ldots, \gamma_{n}\right) \in \mathbb{R}_{+}^{n}$ and $f_{i} \in \mathcal{A}_{p}$ for all $i=\{1,2, \ldots, n\}, n \in \mathbb{N}$. Then $F_{q}(z): \mathcal{A}_{p}^{n} \rightarrow \mathcal{A}_{p}$ is defined as

$$
F_{q}(z)=\mathcal{F}_{q, p, \lambda}^{\delta, \gamma, m}\left(f_{1}, f_{2}, \ldots, f_{n}\right)(z)=\int_{0}^{z}[p]_{q} t^{p-1} \prod_{i=1}^{n}\left(\frac{\mathscr{D}_{q, p, \lambda}^{\delta, m_{i}} f_{i}(t)}{t^{p}}\right)^{\gamma_{i}} d_{q} t
$$

and $G_{q}(z): \mathcal{A}_{p}^{n} \rightarrow \mathcal{A}_{p}$ is defined as

$$
G_{q}(z)=\mathcal{G}_{q, p, \lambda}^{\delta, \gamma, m}\left(f_{1}, f_{2}, \ldots, f_{n}\right)(z)=\int_{0}^{z}[p]_{q} t^{p-1} \prod_{i=1}^{n}\left(\frac{D_{q}\left(\mathscr{D}_{q, p, \lambda}^{\delta, m_{i}} f_{i}(t)\right)}{[p]_{q} t^{p-1}}\right)^{\gamma_{i}} d_{q} t
$$

where $\mathscr{D}_{q, p, \lambda}^{\delta, m_{i}} f_{i}(t)$ is given by (1.5).

It is interesting to observe that several well known and new integral operators are special cases of the operators $F_{q}(z)$ and $G_{q}(z)$. We list a few of them in the following remarks.

Remark 1.2. Letting $m_{i}=0$ for all $i=\{1,2, \ldots, n\}$ and $q \rightarrow 1$, the $q$-integral operator $F_{q}(z)$ reduces to the operator $F_{p}(z)$ studied by B. A. Frasin in [11]. Upon setting $p=1, \delta=0, \lambda=1$ and $q \rightarrow 1$, we obtain the integral operator $D^{k} F(z)$ studied by D. Breaz et al. in [6]. For $p=1, m_{1}=m_{2}=\cdots=m_{n}=0$ and $q \rightarrow 1$, the operator $F_{q}(z)$ reduces to the operator $F_{n}(z)$ which was studied by D. Breaz and N. Breaz in [4]. Observe that when $p=n=1, m_{1}=0, \gamma_{1}=\gamma$ and $q \rightarrow 1$, we obtain the integral operator $I_{\gamma}(f)(z)$ studied by V. Pescar and S. Owa in [15]. Also, for $p=n=1, m_{1}=0, \gamma_{1}=1$ and $q \rightarrow 1$, the $q$-integral operator $F_{q}(z)$ reduces to the Alexander integral operator $I(f)(z)$ studied in [1].

Remark 1.3. Letting $m_{i}=0$ for all $i=\{1,2, \ldots, n\}$ and $q \rightarrow 1$, the $q$-integral operator $G_{q}(z)$ reduces to the operator $G_{p}(z)$ studied by B. A. Frasin in [11]. For $p=1, m_{1}=m_{2}=\cdots=m_{n}=0$ and $q \rightarrow 1$, the operator $G_{q}(z)$ reduces to the operator $G_{\gamma_{1}, \gamma_{2}, \ldots, \gamma_{n}}(z)$ which was studied by D. Breaz et al. (see [7]). Also, for $p=n=1, m_{1}=0, \gamma_{1}=1$ and $q \rightarrow 1$, the $q$-integral operator $G_{q}(z)$ reduces to the integral operator $G(z)$ introduced and studied by J. A. Pfaltzgraff (see $[16])$. 
In the next sections, we study some properties of the $q$-integral operators $F_{q}(z)$ and $G_{q}(z)$ defined by (1.8) and (1.9) respectively, on the classes $\mathcal{S}_{q, p, \lambda}^{\delta, m}(b, \alpha)$ and $\mathcal{C}_{q, p, \lambda}^{\delta, m}(b, \alpha)$. As special cases, the order of convexity of the operators $\int_{0}^{z}\left(\frac{f(t)}{t}\right)^{\gamma} d t$ and $\int_{0}^{z}\left(f^{\prime}(t)\right)^{\gamma} d t$ are also given.

\section{Sufficient conditions for the operator $F_{q}(z)$}

Theorem 2.1. Let $m=\left(m_{1}, m_{2}, \ldots, m_{n}\right) \in \mathbb{N}_{0}^{n}, \gamma=\left(\gamma_{1}, \gamma_{2}, \ldots, \gamma_{n}\right) \in$ $\mathbb{R}_{+}^{n}, 0 \leq \alpha_{i}<p, b \in \mathbb{C}-\{0\}$ and $f_{i} \in \mathcal{S}_{q, p, \lambda}^{\delta, m_{i}}\left(b, \alpha_{i}\right)$ for all $i=\{1,2, \ldots, n\}, n \in$ N. If

$$
0 \leq[p]_{q}+\sum_{i=1}^{n} \gamma_{i}\left(\alpha_{i}-[p]_{q}\right)+\frac{\Re\{b\}}{|b|^{2}}\left(p-1+\left([p]_{q}-p\right) \sum_{i=1}^{n} \gamma_{i}\right)<p
$$

then the q-integral operator $F_{q}$ defined by $(1.8)$ is in the class $\mathcal{C}_{q, p}(b, \eta)$, where

$$
\eta=[p]_{q}+\sum_{i=1}^{n} \gamma_{i}\left(\alpha_{i}-[p]_{q}\right)+\frac{\Re\{b\}}{|b|^{2}}\left(p-1+\left([p]_{q}-p\right) \sum_{i=1}^{n} \gamma_{i}\right) .
$$

Proof. From (1.8), we observe that $F_{q}(z) \in \mathcal{A}_{p}$. On the other hand, it is easy to verify that

$$
D_{q}\left(F_{q}(z)\right)=[p]_{q} z^{p-1} \prod_{i=1}^{n}\left(\frac{\mathscr{D}_{q, p, \lambda}^{\delta, m_{i}} f_{i}(z)}{z^{p}}\right)^{\gamma_{i}} .
$$

Now by logarithmic $q$-differentiation we have,

$$
\frac{\ln q}{q-1} \frac{D_{q}^{2}\left(F_{q}(z)\right)}{D_{q}\left(F_{q}(z)\right)}=\frac{\ln q}{q-1}\left[\frac{p-1}{z}+\sum_{i=1}^{n} \gamma_{i}\left(\frac{D_{q}\left(\mathscr{D}_{q, p, \lambda}^{\delta, m_{i}} f_{i}(z)\right)}{\mathscr{D}_{q, p, \lambda}^{\delta, m_{i}} f_{i}(z)}-\frac{p}{z}\right)\right] .
$$

Therefore,

$$
\frac{z D_{q}^{2}\left(F_{q}(z)\right)}{D_{q}\left(F_{q}(z)\right)}=p-1+\sum_{i=1}^{n} \gamma_{i}\left(\frac{z D_{q}\left(\mathscr{D}_{q, p, \lambda}^{\delta, m_{i}} f_{i}(z)\right)}{\mathscr{D}_{q, p, \lambda}^{\delta, m_{i}} f_{i}(z)}-p\right) .
$$

Multiplying the above equality with $1 / b$, we have

$$
\frac{1}{b} \frac{z D_{q}^{2}\left(F_{q}(z)\right)}{D_{q}\left(F_{q}(z)\right)}=\frac{p-1}{b}+\sum_{i=1}^{n} \gamma_{i} \frac{1}{b}\left(\frac{z D_{q}\left(\mathscr{D}_{q, p, \lambda}^{\delta, m_{i}} f_{i}(z)\right)}{\mathscr{D}_{q, p, \lambda}^{\delta, m_{i}} f_{i}(z)}-[p]_{q}\right)+\frac{[p]_{q}-p}{b} \sum_{i=1}^{n} \gamma_{i}
$$

The above relation is equivalent to

$$
\begin{aligned}
{[p]_{q}+\frac{1}{b} \frac{z D_{q}^{2}\left(F_{q}(z)\right)}{D_{q}\left(F_{q}(z)\right)}=[p]_{q}+\frac{p-1}{b} } & +\sum_{i=1}^{n} \gamma_{i}\left\{[p]_{q}+\frac{1}{b}\left(\frac{z D_{q}\left(\mathscr{D}_{q, p, \lambda}^{\delta, m_{i}} f_{i}(z)\right)}{\mathscr{D}_{q, p, \lambda}^{\delta, m_{i}} f_{i}(z)}-[p]_{q}\right)\right\} \\
& +\left(\frac{[p]_{q}-p}{b}-[p]_{q}\right) \sum_{i=1}^{n} \gamma_{i} .
\end{aligned}
$$


Since $f_{i} \in \mathcal{S}_{q, p, \lambda}^{\delta, m_{i}}\left(b, \alpha_{i}\right)$ for all $i=\{1,2, \ldots, n\}$, we get

$$
\begin{aligned}
& \Re\left\{[p]_{q}+\frac{1}{b} \frac{z D_{q}^{2}\left(F_{q}(z)\right)}{D_{q}\left(F_{q}(z)\right)}\right\} \\
= & {[p]_{q}+\sum_{i=1}^{n} \gamma_{i} \Re\left\{[p]_{q}+\frac{1}{b}\left(\frac{z D_{q}\left(\mathscr{D}_{q, p, \lambda}^{\delta, m_{i}} f_{i}(z)\right)}{\mathscr{D}_{q, p, \lambda}^{\delta, m_{i}} f_{i}(z)}-[p]_{q}\right)\right\} } \\
& -[p]_{q} \sum_{i=1}^{n} \gamma_{i}+\frac{\Re\{b\}}{|b|^{2}}\left(p-1+\left([p]_{q}-p\right) \sum_{i=1}^{n} \gamma_{i}\right) \\
> & {[p]_{q}+\sum_{i=1}^{n} \gamma_{i} \alpha_{i}-[p]_{q} \sum_{i=1}^{n} \gamma_{i}+\frac{\Re\{b\}}{|b|^{2}}\left(p-1+\left([p]_{q}-p\right) \sum_{i=1}^{n} \gamma_{i}\right) } \\
= & {[p]_{q}+\sum_{i=1}^{n} \gamma_{i}\left(\alpha_{i}-[p]_{q}\right)+\frac{\Re\{b\}}{|b|^{2}}\left(p-1+\left([p]_{q}-p\right) \sum_{i=1}^{n} \gamma_{i}\right) . }
\end{aligned}
$$

So, $F_{q}(z) \in \mathcal{C}_{q, p}(b, \eta)$, with $\eta=[p]_{q}+\sum_{i=1}^{n} \gamma_{i}\left(\alpha_{i}-[p]_{q}\right)+\frac{\Re\{b\}}{|b|^{2}}\left(p-1+\left([p]_{q}-\right.\right.$ p) $\left.\sum_{i=1}^{n} \gamma_{i}\right)$.

Taking $p=1, b=1$ and $m_{i}=0$ for all $i=\{1,2, \ldots, n\}$ in Theorem 2.1 we have the following corollary.

Corollary 2.2. Let $\gamma=\left(\gamma_{1}, \gamma_{2}, \ldots, \gamma_{n}\right) \in \mathbb{R}_{+}^{n}, 0 \leq \alpha_{i}<1$ and $f_{i} \in \mathcal{S}_{q}\left(\alpha_{i}\right)$ for all $i=\{1,2, \ldots, n\}, n \in \mathbb{N}$. If

$$
0 \leq 1+\sum_{i=1}^{n} \gamma_{i}\left(\alpha_{i}-1\right)<1
$$

then the q-integral operator

$$
\mathcal{F}_{q}^{\gamma}(z)=\int_{0}^{z}\left(\frac{f_{1}(t)}{t}\right)^{\gamma_{1}} \cdots\left(\frac{f_{n}(t)}{t}\right)^{\gamma_{n}} d_{q} t
$$

is convex with respect to $q$-differentiation of order $\eta$, where $\eta=1+\sum_{i=1}^{n} \gamma_{i}\left(\alpha_{i}-\right.$ $1)$.

\section{Sufficient conditions for the operator $G_{q}(z)$}

Theorem 3.1. Let $m=\left(m_{1}, m_{2}, \ldots, m_{n}\right) \in \mathbb{N}_{0}^{n}, \gamma=\left(\gamma_{1}, \gamma_{2}, \ldots, \gamma_{n}\right) \in$ $\mathbb{R}_{+}^{n}, 0 \leq \alpha_{i}<p, b \in \mathbb{C}-\{0\}$ and $f_{i} \in \mathcal{C}_{q, p, \lambda}^{\delta, m_{i}}\left(b, \alpha_{i}\right)$ for all $i=\{1,2, \ldots, n\}, n \in$ N. If

$$
0 \leq\left([p]_{q}+\frac{(p-1) \Re\{b\}}{|b|^{2}}\right)\left(1-\sum_{i=1}^{n} \gamma_{i}\right)+\sum_{i=1}^{n} \gamma_{i} \alpha_{i}<p
$$

then the q-integral operator $G_{q}$ defined by $(1.9)$ is in the class $\mathcal{C}_{q, p}(b, \eta)$, where

$$
\eta=\left([p]_{q}+\frac{(p-1) \Re\{b\}}{|b|^{2}}\right)\left(1-\sum_{i=1}^{n} \gamma_{i}\right)+\sum_{i=1}^{n} \gamma_{i} \alpha_{i} .
$$


Proof. From (1.9), we observe that $G_{q}(z) \in \mathcal{A}_{p}$. On the other hand, it is easy to verify that

$$
D_{q}\left(G_{q}(z)\right)=[p]_{q} z^{p-1} \prod_{i=1}^{n}\left(\frac{D_{q}\left(\mathscr{D}_{q, p, \lambda}^{\delta, m_{i}} f_{i}(z)\right)}{[p]_{q} z^{p-1}}\right)^{\gamma_{i}} .
$$

Now by logarithmic $q$-differentiation we have,

$$
\frac{\ln q}{q-1} \frac{D_{q}^{2}\left(G_{q}(z)\right)}{D_{q}\left(G_{q}(z)\right)}=\frac{\ln q}{q-1}\left[\frac{p-1}{z}+\sum_{i=1}^{n} \gamma_{i}\left(\frac{D_{q}^{2}\left(\mathscr{D}_{q, p, \lambda}^{\delta, m_{i}} f_{i}(z)\right)}{D_{q}\left(\mathscr{D}_{q, p, \lambda}^{\delta, m_{i}} f_{i}(z)\right)}-\frac{p-1}{z}\right)\right] .
$$

Therefore,

$$
\frac{z D_{q}^{2}\left(G_{q}(z)\right)}{D_{q}\left(G_{q}(z)\right)}=p-1+\sum_{i=1}^{n} \gamma_{i}\left(\frac{z D_{q}^{2}\left(\mathscr{D}_{q, p, \lambda}^{\delta, m_{i}} f_{i}(z)\right)}{D_{q}\left(\mathscr{D}_{q, p, \lambda}^{\delta, m_{i}} f_{i}(z)\right)}-(p-1)\right) .
$$

Multiplying the above equality with $1 / b$, we have

$$
\frac{1}{b} \frac{z D_{q}^{2}\left(G_{q}(z)\right)}{D_{q}\left(G_{q}(z)\right)}=\frac{p-1}{b}\left(1-\sum_{i=1}^{n} \gamma_{i}\right)+\sum_{i=1}^{n} \gamma_{i} \frac{1}{b}\left(\frac{z D_{q}^{2}\left(\mathscr{D}_{q, p, \lambda}^{\delta, m_{i}} f_{i}(z)\right)}{D_{q}\left(\mathscr{D}_{q, p, \lambda}^{\delta, m_{i}} f_{i}(z)\right)}\right) .
$$

Then the above relation is equivalent to

$$
\begin{aligned}
& {[p]_{q}+\frac{1}{b} \frac{z D_{q}^{2}\left(G_{q}(z)\right)}{D_{q}\left(G_{q}(z)\right)} } \\
= & \left([p]_{q}+\frac{p-1}{b}\right)\left(1-\sum_{i=1}^{n} \gamma_{i}\right)+\sum_{i=1}^{n} \gamma_{i}\left\{[p]_{q}+\frac{1}{b}\left(\frac{z D_{q}^{2}\left(\mathscr{D}_{q, p, \lambda}^{\delta, m_{i}} f_{i}(z)\right)}{D_{q}\left(\mathscr{D}_{q, p, \lambda}^{\delta, m_{i}} f_{i}(z)\right)}\right)\right\} .
\end{aligned}
$$

Since $f_{i} \in \mathcal{C}_{q, p, \lambda}^{\delta, m_{i}}\left(b, \alpha_{i}\right)$ for all $i=\{1,2, \ldots, n\}$, we get

$$
\begin{aligned}
\Re\left\{[p]_{q}+\frac{1}{b} \frac{z D_{q}^{2}\left(G_{q}(z)\right)}{D_{q}\left(G_{q}(z)\right)}\right\}= & \left([p]_{q}+\frac{(p-1) \Re\{b\}}{|b|^{2}}\right)\left(1-\sum_{i=1}^{n} \gamma_{i}\right) \\
& \quad+\sum_{i=1}^{n} \gamma_{i} \Re\left\{[p]_{q}+\frac{1}{b}\left(\frac{z D_{q}^{2}\left(\mathscr{D}_{q, p, \lambda}^{\delta, m_{i}} f_{i}(z)\right)}{D_{q}\left(\mathscr{D}_{q, p, \lambda}^{\delta, m_{i}} f_{i}(z)\right)}\right)\right\} \\
> & \left([p]_{q}+\frac{(p-1) \Re\{b\}}{|b|^{2}}\right)\left(1-\sum_{i=1}^{n} \gamma_{i}\right)+\sum_{i=1}^{n} \gamma_{i} \alpha_{i} .
\end{aligned}
$$

So, $G_{q}(z) \in \mathcal{C}_{q, p}(b, \eta)$, with $\eta=\left([p]_{q}+\frac{(p-1) \Re\{b\}}{|b|^{2}}\right)\left(1-\sum_{i=1}^{n} \gamma_{i}\right)+\sum_{i=1}^{n} \gamma_{i} \alpha_{i}$.

Taking $p=1, b=1$ and $m_{i}=0$ for all $i=\{1,2, \ldots, n\}$ in Theorem 2.1 we have the following corollary. 
Corollary 3.2. Let $\gamma=\left(\gamma_{1}, \gamma_{2}, \ldots, \gamma_{n}\right) \in \mathbb{R}_{+}^{n}, 0 \leq \alpha_{i}<p$, and $f_{i} \in \mathcal{C}_{q}\left(\alpha_{i}\right)$ for all $i=\{1,2, \ldots, n\}, n \in \mathbb{N}$. If

$$
0 \leq 1+\sum_{i=1}^{n} \gamma_{i}\left(\alpha_{i}-1\right)<1
$$

then the q-integral operator

$$
\mathcal{G}_{q}^{\gamma}(z)=\int_{0}^{z}\left(D_{q} f_{1}(t)\right)^{\gamma_{1}} \cdots\left(D_{q} f_{n}(t)\right)^{\gamma_{n}} d_{q} t
$$

is convex with respect to $q$-differentiation of order $\eta$, where $\eta=1+\sum_{i=1}^{n} \gamma_{i}\left(\alpha_{i}-\right.$ $1)$.

\section{Applications to convexity and spirallikeness}

As remarked in Section 1, for particular values of the parameters, the classes $\mathcal{S}_{q, p, \lambda}^{\delta, m}(b, \alpha), \mathcal{C}_{q, p, \lambda}^{\delta, m}(b, \alpha)$ reduce to many new classes of analytic functions. If we set $b=e^{-i \beta} \cos \beta(|\beta|<\pi / 2)$ in (1.6) and (1.7), then we have the classes

$$
\mathcal{S}_{q, p, \lambda}^{\delta, m}(\beta, \alpha)=\left\{f \in \mathcal{A}_{p}: \Re\left(e^{i \beta} \frac{z D_{q}\left(\mathscr{D}_{q, p, \lambda}^{\delta, m} f(z)\right)}{\mathscr{D}_{q, p, \lambda}^{\delta, m} f(z)}\right)>\alpha \cos \beta, 0 \leq \alpha<p\right\}
$$

and

$$
\begin{aligned}
& \mathcal{C}_{q, p, \lambda}^{\delta, m}(\beta, \alpha) \\
= & \left\{f \in \mathcal{A}_{p}: \Re\left\{e^{i \beta}\left([p]_{q}+\frac{z D_{q}^{2}\left(\mathscr{D}_{q, p, \lambda}^{\delta, m} f(z)\right)}{D_{q}\left(\mathscr{D}_{q, p, \lambda}^{\delta, m} f(z)\right)}\right)\right\}>\alpha \cos \beta, 0 \leq \alpha<p\right\}
\end{aligned}
$$

respectively. Also, we put

$$
\mathcal{S}_{q, p, \lambda}^{\delta, 0}(\beta, \alpha) \equiv \mathcal{S}_{q, p}(\beta, \alpha) \quad \text { and } \quad \mathcal{C}_{q, p, \lambda}^{\delta, 0}(\beta, \alpha) \equiv \mathcal{C}_{q, p}(\beta, \alpha) .
$$

In particular, we set

$$
\mathcal{S}_{1,1, \lambda}^{\delta, 0}(\beta, \alpha) \equiv \mathcal{S}_{\alpha}^{\beta},(|\beta|<\pi / 2,0 \leq \alpha<1)
$$

and

$$
\mathcal{C}_{1,1, \lambda}^{\delta, 0}(\beta, \alpha) \equiv \mathcal{C}_{\alpha}^{\beta},(|\beta|<\pi / 2,0 \leq \alpha<1)
$$

for the class of $\beta$-spirallike functions of order $\alpha$ (see [13]) and the class of $\beta$-Robertson functions of order $\alpha$ (see [9]), respectively.

In view of Theorem 2.1, Theorem 3.1 and the above definitions, we have the following results. 
Theorem 4.1. Let $m \in \mathbb{N}_{0}, \gamma>0,0 \leq \alpha<p, b \in \mathbb{C}-\{0\}$ and $f \in \mathcal{S}_{q, p, \lambda}^{\delta, m}(b, \alpha)$. If

$$
0 \leq[p]_{q}+\gamma\left(\alpha-[p]_{q}\right)+\frac{\Re\{b\}}{|b|^{2}}\left[p-1+\left([p]_{q}-p\right) \gamma\right]<p,
$$

then the q-integral operator $\mathcal{F}_{q, p, \lambda}^{\delta, \gamma, m}$, defined by

$$
\mathcal{F}_{q, p, \lambda}^{\delta, \gamma} f(z)=\int_{0}^{z}[p]_{q} t^{p-1}\left(\frac{\mathscr{D}_{q, p, \lambda}^{\delta, m} f(t)}{t^{p}}\right)^{\gamma} d_{q} t
$$

is $p$-valently convex of complex order $b$ and type $\eta$ with respect to $q$-differentiation, where

$$
\eta=[p]_{q}+\gamma\left(\alpha-[p]_{q}\right)+\frac{\Re\{b\}}{|b|^{2}}\left[p-1+\left([p]_{q}-p\right) \gamma\right] .
$$

Proof. From (1.8) and (2.2) with $n=1, m_{1}=m, \gamma_{1}=\gamma, \alpha_{1}=\alpha, f_{1}=f$, we get the desired result.

Letting $q \rightarrow 1, p=1$ and $m=0$ in Theorem 4.1, we have:

Corollary 4.2. Let $\gamma>0, \alpha$ be a real number with $0 \leq \alpha<1$. Also, let $f(z)$ be starlike of complex order $b(b \in \mathbb{C}-\{0\})$ and type $\alpha$. If $0 \leq 1+\gamma(\alpha-1)<1$, then the integral operator $\int_{0}^{z}\left(\frac{f(t)}{t}\right)^{\gamma} d t$ is convex of complex order $b$ and type $1+\gamma(\alpha-1)$ in $\mathcal{U}$.

Theorem 4.3. Let $m=\left(m_{1}, m_{2}, \ldots, m_{n}\right) \in \mathbb{N}_{0}^{n}, \gamma=\left(\gamma_{1}, \gamma_{2}, \ldots, \gamma_{n}\right) \in$ $\mathbb{R}_{+}^{n}, 0 \leq \alpha_{i}<p,|\beta|<\pi / 2$ and $f_{i} \in \mathcal{S}_{q, p, \lambda}^{\delta, m_{i}}\left(\beta, \alpha_{i}\right)$ for all $i=\{1,2, \ldots, n\}, n \in$ N. If

$$
0 \leq[p]_{q}+p-1+\sum_{i=1}^{n} \gamma_{i}\left(\alpha_{i}-p\right)<p,
$$

then the $q$-integral operator $F_{q}$ defined by $(1.8)$ is in the class $\mathcal{C}_{q, p}(\beta, \eta)$, where

$$
\eta=[p]_{q}+p-1+\sum_{i=1}^{n} \gamma_{i}\left(\alpha_{i}-p\right)
$$

Proof. From (2.1) with $b=e^{-i \beta} \cos \beta,(|\beta|<\pi / 2)$ and also from (4.1) and (4.2), we obtain the desired result.

Letting $q \rightarrow 1, p=1$ and $m_{i}=0$ for all $i=\{1,2, \ldots, n\}$ in Theorem 4.3 , we have:

Corollary 4.4. Let $\gamma=\left(\gamma_{1}, \gamma_{2}, \ldots, \gamma_{n}\right) \in \mathbb{R}_{+}^{n}$ and $f_{i} \in \mathcal{S}_{\alpha_{i}}^{\beta},(|\beta|<\pi / 2,0 \leq$ $\alpha_{i}<1$ ) for all $i=\{1,2, \ldots, n\}$. If $0 \leq 1+\sum_{i=1}^{n} \gamma_{i}\left(\alpha_{i}-1\right)<1$, then integral operator

$$
F_{\gamma_{1}, \ldots, \gamma_{n}}(z)=\int_{0}^{z}\left(\frac{f_{1}(t)}{t}\right)^{\gamma_{1}} \cdots\left(\frac{f_{n}(t)}{t}\right)^{\gamma_{n}} d t
$$

is in the class of $\beta$-Robertson functions of order $\eta$ where $\eta=1+\sum_{i=1}^{n} \gamma_{i}\left(\alpha_{i}-1\right)$. 
Theorem 4.5. Let $m \in \mathbb{N}_{0}, \gamma>0,0 \leq \alpha<p, b \in \mathbb{C}-\{0\}$ and $f \in \mathcal{C}_{q, p, \lambda}^{\delta, m}(b, \alpha)$. If

$$
0 \leq \gamma \alpha+(1-\gamma)\left([p]_{q}+\frac{(p-1) \Re\{b\}}{|b|^{2}}\right)<p,
$$

then the q-integral operator $\mathcal{G}_{q, p, \lambda}^{\delta, \gamma, m}$ defined by

$$
\mathcal{G}_{q, p, \lambda}^{\delta, \gamma, m} f(z)=\int_{0}^{z}[p]_{q} t^{p-1}\left(\frac{D_{q}\left(\mathscr{D}_{q, p, \lambda}^{\delta, m} f(t)\right)}{[p]_{q} t^{p-1}}\right)^{\gamma} d_{q} t
$$

is p-valently convex of complex order $b$ and type $\eta$ with respect to $q$-differentiation, where

$$
\eta=\gamma \alpha+(1-\gamma)\left([p]_{q}+\frac{(p-1) \Re\{b\}}{|b|^{2}}\right) .
$$

Proof. From (1.9) and (3.2) with $n=1, m_{1}=m, \gamma_{1}=\gamma, \alpha_{1}=\alpha, f_{1}=f$, we get the desired result.

Letting $q \rightarrow 1, p=1$ and $m=0$ in Theorem 4.5, we have:

Corollary 4.6. Let $\gamma>0, \alpha$ be a real number with $0 \leq \alpha<1$. Also, let $f(z)$ be convex of complex order $b(b \in \mathbb{C}-\{0\})$ and type $\alpha$. If $0 \leq 1+\gamma(\alpha-1)<1$, then the integral operator $\int_{0}^{z}\left(f^{\prime}(t)\right)^{\gamma} d t$ is convex of complex order $b$ and type $1+\gamma(\alpha-1)$ in $\mathcal{U}$.

Theorem 4.7. Let $m=\left(m_{1}, m_{2}, \ldots, m_{n}\right) \in \mathbb{N}_{0}^{n}, \gamma=\left(\gamma_{1}, \gamma_{2}, \ldots, \gamma_{n}\right) \in$ $\mathbb{R}_{+}^{n}, 0 \leq \alpha_{i}<p,|\beta|<\pi / 2$ and $f_{i} \in \mathcal{C}_{q, p, \lambda}^{\delta, m_{i}}\left(\beta, \alpha_{i}\right)$ for all $i=\{1,2, \ldots, n\}, n \in$ N. If

$$
0 \leq[p]_{q}+p-1+\sum_{i=1}^{n} \gamma_{i}\left(\alpha_{i}-[p]_{q}-p+1\right)<p,
$$

then the $q$-integral operator $G_{q}$ defined by $(1.9)$ is in the class $\mathcal{C}_{q, p}(\beta, \eta)$, where

$$
\eta=[p]_{q}+p-1+\sum_{i=1}^{n} \gamma_{i}\left(\alpha_{i}-[p]_{q}-p+1\right) .
$$

Proof. From (3.1) with $b=e^{-i \beta} \cos \beta,(|\beta|<\pi / 2)$ and also from (4.2), we obtain the desired result.

Letting $q \rightarrow 1, p=1$ and $m_{i}=0$ for all $i=\{1,2, \ldots, n\}$ in Theorem 4.7, we have:

Corollary 4.8. Let $\gamma=\left(\gamma_{1}, \gamma_{2}, \ldots, \gamma_{n}\right) \in \mathbb{R}_{+}^{n}$ and $f_{i} \in \mathcal{C}_{\alpha_{i}}^{\beta},(|\beta|<\pi / 2,0 \leq$ $\alpha_{i}<1$ ) for all $i=\{1,2, \ldots, n\}$. If $0 \leq 1+\sum_{i=1}^{n} \gamma_{i}\left(\alpha_{i}-1\right)<1$, then integral operator

$$
G_{\gamma_{1}, \ldots, \gamma_{n}}(z)=\int_{0}^{z}\left(f_{1}^{\prime}(t)\right)^{\gamma_{1}} \cdots\left(f_{n}^{\prime}(t)\right)^{\gamma_{n}} d t
$$

is in the class of $\beta$-Robertson functions of order $\eta$ where $\eta=1+\sum_{i=1}^{n} \gamma_{i}\left(\alpha_{i}-1\right)$. 
Further, by suitably specializing the parameters in Theorem 2.1 and in Theorem 3.1, one can easily deduce the known results obtained in [3], [5] and [8].

Acknowledgment. The authors thank the referee for his valuable comments which led to the improvement of this article.

\section{References}

[1] J. W. Alexander, Functions which map the interior of the unit circle upon simple regions, Ann. of Math. (2) 17 (1915), no. 1, 12-22.

[2] F. M. Al-Oboudi, On univalent functions defined by a generalized Sălăgean operator, Int. J. Math. Math. Sci. 2004 (2004), no. 25-28, 1429-1436.

[3] D. Breaz, M. K. Aouf, and N. Breaz, Some properties for integral operators on some analytic functions with complex order, Acta Math. Acad. Paedagog. Nyházi. (N.S.) 25 (2009), no. 1, 39-43.

[4] D. Breaz and N. Breaz, Two integral operators, Studia Univ. Babeş-Bolyai Math. 47 (2002), no. 3, 13-19.

[5] D. Breaz and H. Ö. Güney, The integral operator on the classes $\mathscr{S}_{\alpha}^{*}(b)$ and $\mathscr{C}_{\alpha}(b), \mathrm{J}$. Math. Inequal. 2 (2008), no. 1, 97-100.

[6] D. Breaz, H. Ö. Güney, and G. Ş. Sălăgean, A new general integral operator, Tamsui Oxf. J. Math. Sci. 25 (2009), no. 4, 407-414.

[7] D. Breaz, S. Owa, and N. Breaz, A new integral univalent operator, Acta Univ. Apulensis Math. Inform. (2008), no. 16, 11-16.

[8] S. Bulut, A note on the paper of Breaz and Güney, J. Math. Inequal. 2 (2008), no. 4, $549-553$.

[9] P. N. Chichra, Regular functions $f(z)$ for which $z f^{\prime}(z)$ is $\alpha$-spiral-like, Proc. Amer. Math. Soc. 49 (1975), 151-160.

[10] B. A. Frasin, Family of analytic functions of complex order, Acta Math. Acad. Paedagog. Nyházi. (N.S.) 22 (2006), no. 2, 179-191

[11] Convexity of integral operators of p-valent functions, Math. Comput. Modelling 51 (2010), no. 5-6, 601-605.

[12] G. Gasper and M. Rahman, Basic hypergeometric series, Encyclopedia of Mathematics and its Applications, 35, Cambridge Univ. Press, Cambridge, 1990.

[13] R. J. Libera, Univalent $\alpha$-spiral functions, Canad. J. Math. 19 (1967), 449-456.

[14] M. A. Nasr and M. K. Aouf, Starlike function of complex order, J. Natur. Sci. Math. 25 (1985), no. 1, 1-12.

[15] V. Pescar and S. Owa, Sufficient conditions for univalence of certain integral operators, Indian J. Math. 42 (2000), no. 3, 347-351.

[16] J. A. Pfaltzgraff, Univalence of the integral of $f^{\prime}(z)^{\lambda}$, Bull. London Math. Soc. 7 (1975), no. $3,254-256$

[17] S. D. Purohit, A new class of multivalently analytic functions associated with fractional q-calculus operators, Fract. Differ. Calc. 2 (2012), no. 2, 129-138.

[18] S. D. Purohit and R. K. Raina, Certain subclasses of analytic functions associated with fractional q-calculus operators, Math. Scand. 109 (2011), no. 1, 55-70.

[19] - Fractional $q$-calculus and certain subclass of univalent analytic functions, Mathematica 55(78) (2013), no. 1, 62-74.

[20] Some classes of analytic and multivalent functions associated with $q$-derivative operators, Acta Univ. Sapientiae Math. 6 (2014), no. 1, 5-23.

[21] - On a subclass of p-valent analytic functions involving fractional $q$-calculus operators, Kuwait J. Sci. 42 (2015), no. 1, 1-15.

[22] G. Ş. Sălăgean, Subclasses of univalent functions, in Complex analysisfifth RomanianFinnish seminar, Part 1 (Bucharest, 1981), 362-372, Lecture Notes in Math., 1013, Springer, Berlin, 1983. 
1818 SUNIL DUTT PUROHIT AND KUPPATHAI APPASAMY SELVAKUMARAN

[23] K. A. Selvakumaran, S. D. Purohit, A. Secer, and M. Bayram, Convexity of certain q-integral operators of p-valent functions, Abstr. Appl. Anal. 2014 (2014), Article ID 925902, 7 pp.

[24] P. Wiatrowski, The coefficients of a certain family of holomorphic functions, Zeszyty Nauk. Uniw. Łódz. Nauki Mat. Przyrod. Ser. II No. 39 Mat. (1971), 75-85.

Sunil DutT Purohit

Department of HEAS (Mathematics)

Rajasthan Technical University

Kota - 324010, RAJASTHAN, India

E-mail address: sunil_a_purohit@yahoo.com

Kuppathai Appasamy Selvakumaran

Department of Mathematics

R. M. K College of EngG. and Technology

Puduvoyal - 601206, Tamil Nadu, India

E-mail address: selvaa1826@gmail.com 